Review of Verena Mayer, Christopher Erhard, Marisa Scherini (Eds.): Die Aktualität Husserls

Szanto, Thomas

Published in:

Husserl Studies

DOI:

10.1007/s10743-013-9136-7

Publication date:

2014

Document version

Peer-review version

Citation for published version (APA):

Szanto, T. (2014). Review of Verena Mayer, Christopher Erhard, Marisa Scherini (Eds.): Die Aktualität Husserls. Husserl Studies, 30 (1), 77-88. https://doi.org/10.1007/s10743-013-9136-7 


\section{Verena Mayer, Christopher Erhard, Marisa Scherini (Hg.): Die Aktualität Husserls}

\section{Thomas Szanto}

\section{Husserl Studies}

ISSN 0167-9848

Volume 30

Number 1

Husserl Stud (2014) 30:77-88

DOI 10.1007/s10743-013-9136-7

\section{HUSSERL STUDIES}


Your article is protected by copyright and all rights are held exclusively by Springer Science +Business Media Dordrecht. This e-offprint is for personal use only and shall not be selfarchived in electronic repositories. If you wish to self-archive your article, please use the accepted manuscript version for posting on your own website. You may further deposit the accepted manuscript version in any repository, provided it is only made publicly available 12 months after official publication or later and provided acknowledgement is given to the original source of publication and a link is inserted to the published article on Springer's website. The link must be accompanied by the following text: "The final publication is available at link.springer.com". 


\title{
Verena Mayer, Christopher Erhard, Marisa Scherini (Hg.): Die Aktualität Husserls
}

München, Alber, 2011, 373 Seiten, $€$ 29, ISBN 978-3-495-48462-3

\author{
Thomas Szanto
}

Published online: 3 August 2013

(C) Springer Science+Business Media Dordrecht 2013

Dass Husserl auch im Hundert-Jahres-Jubiläum der Ideen I tatsächlich ,aktuell“ und für eine Reihe gegenwärtiger philosophischer Trends höchst anschlussfähig ist, das verspricht nicht nur der Titel, dies belegen auch eindrücklich die zwölf Aufsätze des vorliegenden Bandes. Das Buch stellt nicht nur eine hervorragende Ergänzung zu dem thematisch stärker fokussierten rezenten Sammelband Husserl und die Philosophie des Geistes (Frank and Weidtmann 2010) dar, zumal auch hier der Anti- bzw. Non-Naturalismus Husserls einen der Leitfäden des Buches bildet; insgesamt handelt es sich dabei in Breite und Tiefe, aber auch was die Qualität der Mehrzahl der Beiträge betrifft, vielmehr um eines der gelungensten der neueren, und nicht nur der deutschsprachigen, Kompendien zu Husserl (vgl. etwa Ierna et al. 2010; Centrone 2013; Embree and Nenon 2013).

Entlang einer klugen editorischen Organisation fügen sich die Beiträge gut in die drei Teile Ontologie, Sprachphilosophie \& Philosophie des Geistes und Handlungstheorie \& Ethik ein (die auch glücklicherweise durch ein brauchbares Sachund Personenregister ergänzt werden). Die Aufsätze decken damit weitgehend die Hauptgebiete aktueller philosophischer Forschung ab. Es spricht auch für die Qualität der editorischen Arbeit, dass sich zahlreiche Beiträge entweder direkt ergänzend oder weiterführend oder aber als Kontrapunkte auf ähnliche Aspekte des Husserl'schen Werks fokussieren. So ist neben dem (Anti-)Naturalismus ein sich mehrfach wiederholendes Motiv etwa Husserls Unterscheidung zwischen Kausalität und Motivation oder jene zwischen objektivierenden und nicht-objektivierenden Akten, Unterscheidungen, die gewichtige Implikationen sowohl für Husserls theoretische als auch praktische Philosophie haben (vgl. die Beiträge von Mayer, Rinofner-Kreidl, Peucker, Loidolt, Vongehr), oder auch Husserls Alternativstellung vis-à-vis der (geisttheoretischen, semantischen oder metaethischen) Internalismus/

T. Szanto $(\bowtie)$

Institute of Philosophy, University of Vienna, Universitätsstraße 7,

1010 Vienna, Austria

e-mail: thomas.szanto@univie.ac.at 
Externalismus-Debatte (vgl. Beyer, Erhard, Rinofner-Kreidl). Gleichwohl ist in diesem Zusammenhang kritisch anzumerken, dass die, ohnehin gegebene, innere Kohärenz des Bandes noch erheblich davon profitiert hätte, wenn sich die AutorInnen stärker mit den Positionen und Argumenten der jeweils anderen auseinandergesetzt hätten, was leider nicht einmal dann geschieht, wenn die Beitragenden komplementäre oder direkt entgegengesetzte Interpretationen vertreten. Warum finden sich etwa keine Kreuzverweis in den Beiträgen von Beyer, Mayer, Erhard und Sowa, oder in den Ethik- und Handlungstheorie-Beiträgen? Wo denn überhaupt solche auftauchen, wird auf persönliche Diskussionen zu den in den jeweiligen Aufsätzen behandelten Themen oder auf anderswo publizierte Vorfassungen der Beiträge hingewiesen, was umso mehr erstaunt, als der Band auf einer gemeinsamen Tagung, die 2009 in München stattfand, basiert. Dieser Mangel an wechselseitigen Referenzen wird jedenfalls größtenteils durch die Qualität der einzelnen Beiträge aufgewogen.

Ein nicht geringer Vorzug des Bandes gegenüber Konkurrenzkompendien liegt auch in der Subtilität und Nachdrücklichkeit, mit der genuin ontologische Aspekte von Husserls Phänomenologie behandelt werden. Diese werden nicht nur im I. Teil (Ontologie), und nicht nur bezüglich der ohnehin in diesem Kontext häufig behandelten Aspekte (wie etwa des Naturalismus), sondern auch mit Bezug auf sozial-ontologische oder sprachphilosophische Probleme berücksichtigt.

So bildet mit seiner differenzierten, distinktiv ontologischen Diskussion von Husserls Verhältnis zum Naturalismus Meixners Beitrag „Natural Psyche: Husserl über die Seele als Naturobjekt" einen gleichsam programmatischen Auftakt. Programmatisch ist er nicht zuletzt deshalb, weil er (wie auch Mayer und RinofnerKreidl) zeigt, dass die transzendentale Phänomenologie entgegen der herkömmlichen, meist aus schlichter Unkenntnis verzerrten analytischen, aber auch entgegen der im phänomenologischen Lager vorherrschenden Standardansicht, Husserls Verhältnis zum Naturalismus ein durchaus komplexes ist, und er keineswegs einen kruden (anti-szientistischen) Anti-Naturalismus tout court vertritt. Mehr noch, die Pointe von Meixners Beitrag liegt gerade darin, anhand des heute eher verpönten Seelen-Begriffs zu zeigen, dass Husserl auch eine, und zwar „rechtmäßige“, „,naturalistische Einstellung “ kennt und entsprechend zwischen einer „rechtmäßigen 'Naturalisierung' des Bewusstseins" (Husserl), nämlich im Sinne der naturalen Mundanisierung von Leib und Seele, und einer, reduktivistischen, physikalistischen Naturalisierung des Bewusstseins unterscheidet (29). Vor diesem Hintergrund und auf Grundlage zahlreicher feinkörniger phänomenologisch-ontologischer Unterscheidungen (etwa zwischen „primären“ und „sekundären Natur-Realitäten“, Manifestationen und Dispositionen), argumentiert nun Meixner überzeugend für die durchaus überraschende These, dass für Husserl zwei sich prima facie ausschließende Kernthesen durchaus kompatibel sind: erstens, dass das Seelische vom Materiellen/Physischem wesensverschieden und zweitens jenes von diesem gleichwohl unabtrennbar (27ff.) sei. Diese Kompatibilitätsthese sei letztlich als Ausdruck von Husserls „,naturalem Substanzdualismus per analogiam“, welche Meixner dann von einem herkömmlichen Subtanzdualismus insofern unterscheidet, als Husserl in den Ideen II der Seele materielle Substanzialität nur per Analogie zum materiellen Ding zuschreibt. Denn die Seele sei zwar vom Materiellen 
unabtrennbar, sofern sie aber extensionslos ist (und genau darin besteht ihr Wesensunterschied zum dinglichen Materiellen), ist sie zugleich immateriell. Überzeugend, wenn auch etwas gedrängt und dementsprechend zuweilen schwer nachvollziehbar, ist in Meixners Argumentation der Schritt, den er ausgehend von einer funktionalistisch bestimmten Konzeption der ,naturalen Seele“ hin zur „kausalitätsfundierenden Rolle“ naturaler Dispositionen, verstanden als „kausale Knotenpunkte“, macht (31f.), um dann Husserl einen „funktionalistischen Determinismus" bezüglich der Dispositionen der Seele zuzuschreiben (35). Demnach seien materielle Dinge und eben auch die immaterielle, naturale Seele (dispositionale) Knotenpunkte von Ursache/Wirkungs-Verhältnissen und gleichzeitig solche Verhältnisse fundierend, ohne wohlgemerkt in einen konstitutionstheoretischen Zirkel zu münden. Denn Meixners funktionalistischer Deutung zufolge können für Husserl, anders als etwa für Ryle, naturale Dinge sowohl „(ursächliche) Umstände“ als auch „(wirkungsmäßige) Zustände“ sein, wobei die „geregelte Zusammengehörigkeit“ zwischen diesen dispositionellen Polen durch die Dingeigenschaften selbst konstituiert werde, deren 'Aggregat" bzw. „Dispositionseinheit“, eben nichts anderes als das naturale Ding sei.

Ganz auf der Höhe von Meixners subtilen ontologischen Unterscheidungen unterzieht auch der Beitrag von De Monticelli „Alles Leben ist Stellungnehmen Die Person als praktisches Subjekt" Husserls Theorie der Personalität einer ontologischen und zugleich praktischen Analyse. Mit der überzeugenden Verteidigung ihrer zentralen These, wonach Personalität und personale Identität für Husserl durch genau jenes Subjekt individuiert wird, welchem ,es gelingt, durch seine eigenen Akte“ (im doppelten Sinn, also 'mittels' und 'aus freien Stücken" bzw. 'autonom') ,über die eigenen Zustände zu emergieren“ (41), gelingt es auch der Autorin das Grundgerüst für eine phänomenologische und praktisch fundierte Ontologie von (mentalen) Akten und Zuständen bzw. Handlungen und Ereignissen zu liefern. Interessant wäre hierbei eine Auseinandersetzung mit der gegenwärtigen Debatte um mentale Handlungen gewesen (vgl. O'Brien and Soteriou 2009), insbesondere nachdem De Monticelli eine ,irreführende“ Gleichsetzung von intentionalen Akten (qua Erlebnissen) mit mentalen Akten (qua Handlungen) im Fall von Husserl, zu Recht wohlgemerkt, ablehnt (47ff.). Auch hätten einige analytische handlungstheoretische Modelle (wie etwa H. Frankfurts Theorie von Meta-Volitionen oder M. Bratmans bzw. G. Harmans Theorien von Intentionen als normativ selbstverbindlichen Handlungsplänen) der Autorin Schützenhilfe liefern können (vgl. 52f.). Die durchaus starken Thesen, und insbesondere jene, wonach Personalität fundiert ist in einer Hierarchie von aktiven und autonomen Stellungnahmen zu den eigenen Zuständen und deren „Management“ (50ff.), hätten zuweilen etwas detailliertere Auseinandersetzungen verdient, sie stellen aber letztlich auch in ihrer vorliegenden Form eine gute Basis für eine noch weitgehend ausstehende praktisch-normative phänomenologische Rekonstruktion personaler Identität dar.

Der Beitrag „Husserls intentionale Soziologie“ von Caminada verdient insofern besondere Beachtung, als er einen weitgehend ignorierten Aspekt von Husserls ansonsten vieldiskutierter späterer Theorie der Intersubjektivität, nämlich seine „transzendentale Soziologie“ und die berühmt-berüchtigte Theorie „Personalitäten 
höherer Ordnung“ bzw. eines „Gemeingeistes“, aufgreift und für die KollektiveIntentionalitäts-Debatte fruchtbar macht, welche die gegenwärtige analytische Handlungstheorie und Sozialontologie dominiert. Trotz einiger jüngster Annährungsversuche vonseiten der Phänomenologie (vgl. Chelstrom 2013; Salice and Schmid forthcoming; Moran and Szanto forthcoming) ist ein solcher Dialog umso wichtiger, als man mit Husserl durchaus wichtige kritische Präzisierungen in die Debatte einbringen kann: Die wichtigsten dabei betreffen, wie Caminada treffend zeigt, erstens, die konstitutive Verschränkung jener Komponenten, die in der analytischen Diskussion typischerweise als separate Momente kollektiver Intentionalität konzipiert werden: nämlich Subjekt, Modus, Gehalt und Korrelat kollektiver Intentionen (68f.); zweitens, die in der analytischen Debatte stark vernachlässigten passiven, vorreflexiven, habitualisierten und affektiven, kurz: nonkognitiven Aspekte in der Konstitution von Kollektivität $(68,78 f$.) und die entsprechende genetische Verflechtung individueller Sinnhorizonte; drittens weist Caminada darauf hin, dass wir es bei Husserl (wie auch bei anderen frühen phänomenologischen SozialontologInnen, etwa bei E. Stein, G. Walther oder T. Otaka), je nach der feinkörnigen Art intentionaler Integration bzw. Vergemeinschaftung, mit einer weit komplexeren konstitutiven Schichtenanalyse verschiedener Typen von sozialen Entitäten bzw. Kollektivitäten (,,praktische Willensgemeinschaften“, ,Subjektvielheiten“ etc.) zu tun haben als bei den meisten analytischen AutorInnen, wobei dieser Aspekt bei Caminada leider etwas unterbelichtet bleibt. Wie Caminada jedenfalls richtig zeigt, läuft mithin Husserls genetisch-konstitutive Konzeption $a b$ ovo ,vergemeinschafteter“ Individuen einer Konzeption „,vorsozialer“ Individuen als „Atome“ sozialer Realität zuwider, „die sich erst mittels Vereinbarungen“ (68) à la M. Gilbert vergemeinschafteten. Damit weist er auch überzeugend sowohl den Standardvorwurf des (methodologischen) Individualismus/Solipsismus als auch den, ironischerweise damit zusammenhängenden, Vorwurf des Kollektivismus zurück. So wird Husserl etwa vorgeworfen, aufgrund seiner vermeintlich internalistischen, transzendental-subjektivistischen Analyse intersubjektiver Konstitution nicht in der Lage zu sein, gemeinsame oder geteilte Intentionalität anders $\mathrm{zu}$ fassen als durch eine Projizierung egologischer Strukturen auf Kollektivsubjekte höherer Ordnung. Durch diesen ,einzelsubjektbasierten Begriff des Sozialen“ jedoch würden einerseits Individualsubjekte in ihrem „Verhältnis zur Sozialität depotenziert“, andererseits genuine Kollektivität pace Husserl individualisiert, und damit würde der sui generis-Struktur von WirIntentionalität nicht Rechnung getragen (vgl. Schmid 2000, 24f.). Obwohl Caminada diesem Doppelvorwurf den Wind aus den Segeln nimmt, lässt er die von ihm selbst aufgeworfene und auch von Schmid implizit angemahnte kritische Anfrage an Husserl weitgehend offen, nämlich ob es denn im Rahmen einer intentionalen Sozialontologie „nicht konsequenter wäre, sich vom Paradigma des egologischen Subjekts zu lösen“ (74).

Im II. Teil evaluieren die AutorInnen argumentationsstark und kritisch Husserls Beitrag zur aktuellen Philosophie des Geistes und der Sprachphilosophie, wobei gerade die Überschneidungen zwischen diesen beiden zentralen modernen philosophischen Teilgebieten in Husserls Werk auf sinnfällige Art und Weise kenntlich werden. 
In seinem überaus lesenswerten „Husserl über Begriffe“ widmet sich Beyer einem wenig erforschten Aspekt von Husserls Phänomenologie und nähert sich dem Thema treffenderweise nicht über rein sprachphilosophisch-semantische Überlegungen, sondern über das Husserl in gewisser Weise vertrautere Terrain der Geistphilosophie, genauer über Fodors einflussreicher repräsentationalistische Theorie des Geistes an, in welcher Begriffe als Grundkomponenten intentionaler Zustände gefasst werden. Entlang eines werkhistorischen Querschnitts aus Husserls Überlegungen zu Begriffen, von der Philosophie der Arithmetik über seine von Bolzano beeinflusste Theorie abstrakter und propositionaler Begriffe (Sätze) bis hin zur intersubjektiv-kommunikativen Begriffs-Konzeption der Cartesianischen Meditationen, zeigt Beyer überraschender-, aber auch plausiblerweise, dass Husserls Phänomenologie - seinem intentionalen Anti-Repräsentationalismus zum Trotz - alle Bausteine enthält, um Fodors Desiderata an eine adäquate Begriffs-Konzeption zu erfüllen (d. i. kausale Relationalität; extensionale Anwendbarkeit; repräsentationale und propositionale Funktion; Erlernbarkeit, intersubjektive Kommunizierbarkeit und Überprüfbarkeit; vgl. 89). Neben einer guten kritischen Fodor-Darstellung und einer überaus konzisen Diskussion von Bolzanos Satz- und Vorstellungslehre (99) ist v. a. Beyers Hinweis auf die Ähnlichkeiten der späten Lebenswelt-Theorie Husserls, im Rahmen deren die Begriffs-Konzeption eine distinktiv intersubjektive, ja 'interkulturelle', Prägung erfährt, mit Wittgensteins „Hintergrund“- und „Weltbild“-Konzeption wertvoll (111). Etwas weniger begründet hingegen ist der Verweis auf Husserls ,frühe Version des Externalismus“, zumal das auch in Erhards Artikel ausführlicher diskutierte Husserl'sche Zwillingserde-Gedankenexperiment (auf das ja Beyer selbst als erster hingewiesen und dann en detail evaluiert hat; vgl. Beyer 1996; 2000) hier zumindest ambivalent vis-à-vis dem vermeintlichen Externalismus gedeutet wird. So bemerkt auch Beyer, dass Husserl intentional-gegenständliche Identität wesentlich an die erst-personal erlebnismäßige bzw. phänomenale Identität des jeweils Gegebenen knüpft. Schließlich wäre in diesem Zusammenhang eine Husserl'sche Auseinandersetzung mit der im Anschluss an J. McDowell in analytischen Kreisen zurzeit sehr beliebten Debatte um konzeptuelle versus nicht-konzeptuelle Wahrnehmungsinhalte lohnenswert gewesen, auf die Beyer leider nur in einer Fußnote kurz anspielt (98).

Mayers Beitrag „Husserl und die Kognitionswissenschaften“ liefert eine gute Darstellung der Probleme, mit denen sich eine etwaige ,phänomenologische Kognitionswissenschaft“ (142) zu konfrontieren hat. Mayer hat mit ihrer Konzentration auf drei zentrale naturalistische Modelle (nämlich das lange dominante Computermodell des Geistes, die Kausaltheorie der Referenz und das spezifisch neurophänomenologische Naturalisierungsprogramm à la Varela), und mit einem Exkurs in die Qualia-Debatte, eine gute Strukturierung getroffen, anhand derer sie einerseits konzise in die klassischen kognitionswissenschaftlichen Grundparadigmen einführen, andererseits die dabei aus phänomenologischer Sicht zentralen Problemfelder identifizieren kann. Im Rahmen ihrer Darstellung des Problems der Motivation diskutiert Mayer dann dankenswerterweise nicht nur die üblichen antikausalistischen Argumente, sondern auch die in der Husserl-Forschung bisher eher vernachlässigten, wenn auch etwas ambivalenten Überlegungen Husserls zur damaligen psychophysischen Parallelismus-Debatte bzw. zur Frage nach der 
„Wechselwirkung“ von Motivation und Kausalität in den Ideen II (140f.). Vor diesem Hintergrund lautet Mayers interessante Schlusspointe, dass pace Chalmers \& Co. für Husserl mitnichten die einfachen Empfindungen bzw. die hyletischen Empfindungsdaten (die heutigen Qualia) das hard problem einer naturalistischen Erklärung des Bewusstseins seien. Denn gerade diese ließen sich unter geeigneten methodologischen Adaptionen in einer ,naturalisierten Phänomenologie“ (Petitot et al.) sehr wohl naturalisieren. Was vielmehr einem solchen Projekt phänomenologisch gesehen hartnäckig zu widerstreben scheint, ist gerade jener Aspekt des Bewusstseins, dessen Naturalisierbarkeit etwa Funktionalisten mehr Erfolgsaussichten bescheinigen, nämlich die Sphäre der Motivation als (akausale) Wesensgesetzlichkeit des Bewusstseinslebens.

Gleich zwei Artikel widmen sich der oft ignorierten und genauso oft missverstandenen eidetischen Phänomenologie Husserls, und seiner umstrittenen Wesenslehre bzw. Theorie der Wesensschau, und es ist, wieder einmal, bedauerlich, dass die beiden AutorInnen, bei aller thematischen Nähe, nicht mit den Positionen der jeweils anderen auseinandersetzen, zumal sie gegenläufige Interpretationen vertreten.

Sowa bringt in „Das Allgemeine und das Gemeinsame: Anmerkungen zum Proton Pseudos der Lehre von Husserls Wesensanschauung “ eine historisch äußerst kenntnisreiche und auch systematisch höchst informative Kritik an Husserls Identifizierung logisch-semantischer Allgemeinbegriffe mit einer ontologischen Bestimmung des Wesensbegriffs, als etwas Einzelgegenständen zugrundeliegendes „Gemeinsames“, in Anschlag. Auf Grundlage einer konzisen Darstellung der an Aristoteles anschließenden Bestimmung von Allgemeinbegriffen, vom scholastischen Universalienstreit über die Logik von Port-Royal, Kant und Lotze bis hin zu Husserl, führt er anhand einiger Gegenbeispiele anschaulich vor, dass eine solche Engführung, etwa bei Allgemeinbegriffen mit a priori leeren Extensionen (z. B.: „eine Primzahl zwischen 7 und 11“) versagt. Denn die Funktion von die Funktion von Allgemeinbegriffen besteht, wie hieran ersichtlich wird, nicht darin, mehrere Einzelgegenstände unter ein Gemeinsames zu subsumieren, sondern darin, etwas Allgemeines zu prädizieren, und d. h., eine Funktionsstelle in Aussagen einzunehmen, welche auch wahrheitsfunktional evaluiert werden kann. Mit dieser durch Frege eingeleiteten Wende in der Bestimmung von Allgemeinbegriffen, die Husserl zwar „gekannt“, deren Tragweite er aber eben nicht „erkannt“ hätte (162), verteidigt nun Sowa eine durchaus originelle Rekonstruktion der Husserl'schen „reinen Wesen“ als „reinen propositionalen Funktionen“, deren Werte jedoch, anders als bei Frege, nicht Wahrheitswerte, sondern ontologische „Sachverhaltsfunktionen“ (Werte des Bestehens/Nicht-Bestehens) seien (168f.). Entsprechend würden in der Wesensanschauung bzw. eidetischen Variation nicht Wahrheitsfunktionen evaluiert, sondern Wesensgesetze verifiziert, sich auf die mögliche (intersubjektive) Ausweisung von Sachverhalten beziehen.

So sehr nun die pointierte und historisch informierte Argumentation Sowas auch überzeugt, ist es sinnvoll, sie zusammen mit der nachfolgenden von Mayer zu lesen, welche die rein logisch-semantische Deutung der Husserl'schen Wesenslehre mit dessen genetisch-gegenstandskonstitutiver und nicht zuletzt normativ-erfahrungsleitender Funktion gut ergänzt. So verteidigt Mayer, im Gegensatz zu Sowa, in 
ihrem zweiten Beitrag zu dem Band: „Regeln, Spielräume und das offene Undsoweiter: Die Wesensschau in Erfahrung und Urteil" die originelle Husserl'sche Fassung der Wesensschau als eine eigenständige normative Erkenntnisfunktion, und zwar diesmal mit Blick auf das über Schlick bis dato im analytischen Raum tradierte Fehlurteil, es handle sich dabei um eine Art intuitionistisches SichHineinversetzen in Ideen, das dann in irgendwelchen obskuren privaten Evidenzgefühlen mündete. Demgegenüber interpretiert Mayer richtigerweise die eidetische Variation und ihren Korrelat-Begriff, die Wesensschau, nicht als ein „esoterisches Brimborium“, sondern als das ,gewöhnliche Handwerkszeug der Philosophen“ (189), welches jedoch, anders als rein sprachanalytische Begriffsanalyse, jederzeit an mögliche subjektive Erfahrung (und sei es nur in freier (eidetischer) Phantasievariation) rückgebunden und/oder rückzubinden sei, und auch an dieser sich insofern bewähren müsse, als „empirische“ und auch ,reine“ Allgemeinbegriffe (empirische bzw. reine Wesen) notwendige „Spielräume der Erfahrung“ bereitstellten und sich an diesen epistemisch messen lassen müssten. Damit seien aber, und hier zumindest stimmt Mayer mit Sowa überein, die in der Wesensschau gegebenen Allgemeinheiten nicht nur Kants Begriffskonzeption als „Regeln empirischer Erfahrung“, die einerseits reflexiv aus der Erfahrung gewonnen und anderseits normativ und konstitutiv diese ,bestimmen“, analog (183) (vgl. auch oben zu Beyer); Mayer weist auch auf interessante und überzeugende Parallelen von Husserls Wesensschau und Evidenz-Begriff sowohl mit Russells Begriff der acquaintance als auch mit Wittgensteins Tractatus-Theorie des Zeigens bzw. der „allgemeinen Formenreihen“ und seiner späteren Theorie des Regelfolgens als „wesens-artigem Verfahren in der analytischen Tradition“ hin. Damit wird aber auch das Bild von einem allzu tiefen Bruch zwischen Husserls und analytischen Theorien von Allgemeinbegriffen historisch zurechtgerückt (186f.). Doch während Sowa minutiös und überzeugend nachweist, dass Husserl einem logisch folgenschweren Missverständnis aufliegt, wenn er das begriffliche Allgemeine als ein gegenständliches Gemeinsames auffasst, übernimmt Mayer diese Engführung recht unkritisch, was umso mehr erstaunt, als sie in einigen Fußnoten auf diesbezügliche Diskussionen mit Sowa verweist. Dies erweist sich im Kontext ihrer Diskussionen der Wesenslehre allerdings insofern nicht als gravierend, als Mayer mit ihrer Fokussierung auf die genetischen gegenstandsindividuierenden Aspekte in der Konstitution von Wesensallgemeinheiten (wie dies ja in Erfahrung und Urteil vorliegt), ob gewollt oder ungewollt, eine Korrektur bzw. Ergänzung zu Sowas fregeanisch-wahrheitsfunktionaler Bestimmung begrifflicher Allgemeinheiten vornimmt.

Der Beitrag Erhards „Empirische Bedeutung und Twin Earth - Husserls Bedeutungstheorie modifiziert" kann exemplarisch für eine argumentationsstarke Auseinandersetzung Husserls vor der Folie gegenwärtiger Debatten in der analytischen Tradition stehen und verknüpft in der Zusammenführung von Philosophie des Geistes und Sprachphilosophie die zentralen Problemstellungen, die in den restlichen Beiträgen des II. Teils expliziert werden. Erhards Artikel fügt sich in die in jüngster Zeit wachsende Reihe von Arbeiten, die Husserl vor dem Hintergrund der ausufernden und mittlerweile etwas festgefahrenen semantischen und geisttheoretischen Internalismus/Externalismus-Debatte neu $\mathrm{zu}$ evaluieren 
versuchen, eine Aufgabe, die umso lohnenswerter ist, als hierbei einige Pointen von Husserls transzendentalem Perspektivenwechsel klar konturiert und in zeitgenössischer Terminologie reformuliert werden können. Entsprechend schlägt Erhard, entlang einer guten Diskussion von Husserls komplexer Theorie wesentlich okkasioneller Ausdrücke bzw. seiner oft vernachlässigten, zentralen Unterscheidung von „empirischen“ und „reinen Begriffen“ eine kongeniale Modifikation von Husserls früherer, platonistisch-realistischer Bedeutungstheorie vor: Eine solche modifizierte „Speziestheorie der Bedeutung “ sei nämlich nötig, da die Husserl'sche Speziestheorie mit gravierenden Problemen konfrontiert sei, wenn es um die Erklärung der Semantik okkasioneller (und mithin aller empirischen) Ausdrücke gehe, Probleme, die eben auch Husserls Unterscheidung von „Schwanken der Bedeutungen“" und „Schwanken des Bedeutens" nicht zu lösen vermag (202f.). Erhard argumentiert nun, dass Bedeutungen nicht allein durch kontextunabhängige, reine Spezies festgelegt werden (ob reine Spezies denn überhaupt noch eine bedeutungsdeterminierende Funktion in Erhards Version haben, scheint mir nicht ganz klar zu sein). Vielmehr würden die semantischen Identitätsbedingungen von empirischen Ausdrücken durch intersubjektive, diachron-dynamische und holistische Wahrnehmungs- und Bewährungskontexte, also nicht intra-mental, (ko)determiniert. Dies ist - entgegen Erhards Eigeninterpretation - allerdings keine, zumindest keine herkömmliche, internalistische These. Dass Erhard, zugegebenermaßen an einer qualifizierten (,in gewissem Sinn“; 195) Version des Internalismus festhält, scheint mir mit seiner interpretativen Grundprämisse zusammenzuhängen: So setzt er (wie Beyer) die fregeanisch inspirierte Interpretation der Husserl'schen Bedeutungstheorie voraus, wonach Noemata, analog zu den Spezies von Husserls früherer Bedeutungstheorie, ,ideale Gegenstände sui generis“ (193) seien und bedeutungsverleihende Akte sich „durch“ oder mittels ihrer Bedeutungen (Noemata) auf ihre Gegenstände beziehen. Pace Erhard folgt aus der „'intentionalistischen“ Auffassung von Bedeutung“, wonach Ausdrücke nur bedeutungsvoll sind, wenn sie sich auf Gegenständlichkeiten beziehen, jedoch keineswegs notwendig, dass sich Ausdrücke „'durch" oder 'mittels" " ihrer Bedeutung auf diese Gegenständlichkeiten beziehen (198f.). Diese bekanntlich nicht unumstrittene Interpretation lässt sich auch durch die im Beitrag angeführten Zitate keinesfalls ohne weiteres belegen (vgl. insbes. 199). Sie hat jedenfalls zur Folge, dass man, und auch Erhard, sich gezwungen sieht, mit, gegen oder für Husserl die (Freges'che/Carnap'sche/Putnam'sche) semantische „Determinationsfrage“ (222) beantworten zu müssen, also, ob und wie Intensionen Extensionen bestimmen. Stattdessen wäre es naheliegender gewesen, wie Erhard in den Schlussparagraphen selbst zu Recht betont, Husserls „'transzendentale“ oder 'konstitutive" [Theorie der] Beziehung zwischen Sinn/Bedeutung und Gegenstand" (229) stark zu machen, eine Beziehung, die in Husserls intentionalitätstheoretischer Wendung keineswegs identisch ist mit jener zwischen Intensionen/Bedeutungen und Extensionen/Referenten. Fairerweise muss angemerkt werden, dass sich Erhard dessen sehr wohl bewusst zu sein scheint, wenn er etwa treffend bemerkt, dass ,eine ausführliche [positive] Antwort [auf die Frage, ob Putnams externalistische Determinationsthese, wonach gleiche Bedeutungen gleiche Extensionen implizieren, mit Husserls vermeintlichem Internalismus, demzufolge es „,ür das Verstehen 
eines Ausdrucks genügt, eine entsprechende 'enge" Bedeutungsintention zu vollziehen" (221), kompatibel sei; T. Sz.] letztlich mit Husserls dynamischer Konstitutionstheorie bzw. mit seinem phänomenologischen Idealismus [zusammenfällt]“ (224). Letztlich ist denn auch, sofern man Erhards metatheoretische fregeanische Grundprämisse akzeptiert, seiner Konklusion jedenfalls zuzustimmen, wonach nämlich Intensionen ihre Referenten, unter Berücksichtigung der Horizontintentionalität, intersubjektiv, holistisch und dynamisch festlegen, oder eben besser: konstituieren, und umkehrt, phänomenologisch gesehen, pace Putnam \& Co. keine physikalischen Differenzen in der Welt, die ,in the long run“ erscheinungsmäßig „kollektiv opak sind“, Unterschiede in Bedeutungen festlegen können (228f.).

Im III. Teil des Bandes (Handlungstheorie \& Ethik), haben die HerausgeberInnen eine gute Entscheidung getroffen, Husserls Beitrag zur praktischen Philosophie nicht auf die Ethik zu beschränken. Denn Husserls Handlungstheorie ist, trotz des jüngst vermehrten Interesses an praktischen Aspekten seines Werkes, ein nach wie vor stark vernachlässigtes Gebiet, anhand deren gerade Husserls Verhältnis zum Naturalismus, etwa entlang der Unterscheidung von Kausalität und Motivation schärfere Konturen gewinnt.

So demonstriert Rinofner-Kreidl im Aufsatz „Motive, Gründe und Entscheidungen in Husserls intentionaler Handlungstheorie“, der einen der Höhepunkte des vorliegenden Bandes darstellt, eindrücklich, dass sich mit Husserl nicht nur die mittlerweile klassische inner-analytische Diskussion zwischen kausalen und motivationalen bzw. intentionalen Handlungstheorien kritisch beleuchten, sondern auch eine überzeugende alternative Theorie von Handlungsmotiven skizzieren lässt. Rinofner-Kreidl macht dabei klar, dass eine adäquate Handlungstheorie einer intentional-theoretischen Fundierung bedarf, sofern nämlich gilt, dass ,zwischen Bewusstsein und Handlung kein wesentlicher Hiatus [besteht], der über den Unterschied hinausgeht, dass wir im einen Fall verändernd in Geschehnisabläufe eingreifen, im anderen nicht“ (232). Von da her ist denn auch eine phänomenologische Handlungstheorie nicht auf die Erklärung von temporären Handlungsverläufen im engeren Sinn beschränkt, sondern umfasst, entlang der Analyse der teleologisch-motivationalen Struktur des mentalen Lebens, die kognitive, axiologische, aber auch affektive, emotionale und passive Sphäre des Praktischen, die etwa auch „habitualisierte Motivationen“ einschließt. Phänomenologisch gesehen sind dabei kognitive und nicht-kognitive Handlungsmotive mitnichten als „reine Gegenpole“, sondern vielmehr qua Bewusstseinsleistung einem komplexen „Funktionsganzen“ zugeordnet (272f.) (Hier ergeben sich interessante Parallelen zur Betonung habitualisierter Aspekte im Fall kollektiver Intentionalität im Beitrag von Caminada). Rinofner-Kreidl zeigt damit zugleich den systematischen und metatheoretischen 'Gap' zwischen einer phänomenologischen und dem Mainstream der analytischen Handlungstheorie auf. Einen möglichen Anschluss an gegenwärtige analytische Theorien verdeutlicht die Autorin dann u. a. in ihrer beispielhaften Diskussion der metaethischen Internalismus/Externalismus-Debatte. Sie zeigt Husserls Alternativstellung vis-à-vis akteursneutralen und akteursabhängigen Konzeptionen von Handlungsgründen auf und präsentiert dabei, quasi en passant, alle Bausteine einer eigenständigen phänomenologischen Handlungstheorie (256-262). Angelehnt an J. Wallaces „Volitionalismus“ argumentiert sie schließlich dafür, dass 
rationale Motivation (entgegen internalistischen, aber auch herkömmlichen externalistischen bzw. akteursneutralen Bestimmungen) wesentlich mit normativ gefassten Handlungsgründen und letztlich mit personaler Selbstbestimmung zu tun hat. Die dem 'hermeneutischen Selbsteinschluss' des Akteurs bei der Handlungserklärung und einer ,prozessual verstandenen“ (moralischen) Akteurschaft entsprechende „Dialektik von Selbstentwurf und Selbstmanifestation in der Interpretation von Handlungsgründen" exemplifiziert sie treffend anhand der medizinethischen Informed Consent-Problematik (270f.) und geht damit einen Schritt über Husserl hinaus, was freilich den Ansatz nicht minder überzeugend macht. Indem der Aufsatz zeigt, dass eine phänomenologische Handlungstheorie nicht ohne (meta-)ethische und normative Überlegungen auskommt, bereitet sie damit auch den Boden für die folgenden Aufsätze vor, die sich mit Husserls Ethik im engeren Sinn auseinandersetzen.

Die drei Beiträge, die sich mit Husserls Ethik (Peucker und Loidolt) bzw. seinen Forschungsmanuskripten zur Analyse von Gemüts-, Gefühls- und Willensakten (Vongehr) beschäftigen, gehören zu den am problematischsten des Bandes, was nicht so sehr an der Qualität der Beiträge liegt, sondern vielmehr daran, dass Husserl trotz seiner intensiven Bemühungen ab den 1910er-Jahren weder eine wirklich überzeugende Ethik noch eine systematische, zurzeit vieldiskutierte Theorie der Emotionen vorzuweisen hat. Der engere Fokus dieser Beiträge bringt auch einige allzu starke thematische Überschneidungen sowohl untereinander als auch mit Rinofner-Kreidls Aufsatz mit sich. Dessen ungeachtet bieten die Beiträge einige durchaus interessante Einsichten, insbesondere zu werkgenetischen Binnendifferenzierungen und der Abgrenzung von Husserls praktischer Philosophie von historischen und zeitgenössischen Konkurrenzmodellen.

So zeigt etwa Peucker in „Husserls Ethik zwischen Formalismus und Subjektivismus “ auf, dass Husserl im Streit zwischen Universalismus und Partikularismus und seines transzendentalphilosophischen Pendants (Formalismus/Subjektivismus), einmal mehr, eine Sonderstellung zukommt. Entsprechend argumentiert Husserl, analog zum Anti-Psychologismus der Prolegomena, einerseits gegen gefühlssubjektivistische Relativierungen bzw. Psychologisierungen von Werten (etwa von Hume), andererseits gegen formalistische Begründungen allgemeingültiger moralischer Urteile (à la Kant). Ausgehend von dieser Grundthese zeichnet Peucker detailreich Husserls frühere „formale Axiologie“ und „formale Praktik“, als „Paralleldisziplinen“ zur reinen Logik, nach (281-289), weist auf ungelöste Probleme bezüglich des Zugangs zu objektiven Werten in nicht-objektivierenden axiologischen Gefühlsakten bzw. der mangelnden phänomenologischen Analyse der emotiven Gegebenheitsweise von Werten hin, um dann Husserls spätere, personale Ethik darzulegen. Deren an kant bzw. Fichte (wohlgemerkt nicht an Scheler) orientierter aufklärerischer Personenbegriff bzw. deren ethisches Leitkonzept, das durch radikale und rationale „Selbstkritik“ bzw. einer ominösen „Erneuerung“ der Person zu gewinnende Ideal des „ethischen Lebens“ (294f.), bleiben aber, wie eben bei Husserl auch, leider etwas skizzenhaft.

Einen systematischeren Versuch, Husserls Ethik neu zu evaluieren, nimmt Loidolt vor, deren Beitrag sich entlang einer Reihe von Leitfragen gruppiert: Die erste Frage nach dem Zusammenhang zwischen Ethik und Phänomenologie bzw. deren Weichenstellungen, nimmt einige Motive, die wir schon bei Peucker und 
Rinofner-Kreidl kennengelernt haben, auf. Loidolt beantwortet sie mit Husserls Betonung des transzendental gewendeten Grundmotivs rationaler und personaler Selbstbestimmung, die eben nicht nur formal-axiologische, sondern auch affektive, emotionale, volitionale und insbesondere Sinn-konstitutive Aspekte des Normativen berücksichtigt. Auch bei der Beantwortung der weiteren Fragen nach Wertrealismus/-objektivismus, moralischem Intuitionismus bzw. Instinkten und moralischer Rechtfertigung stehen motivationale und non-kognitive Aspekte der personalistischen Ethik und die Analyse der etwas nebulösen Konzeption einer normativen, „,absoluten Affektion“ (Husserl) im Vordergrund. Um Husserls Ethik im zeitgenössischen Kontext zu verorten, wäre es hier allerdings angebracht gewesen, die aktuellen analytischen Debatten rund um moralischen Realismus/Anti-Realismus bzw. Kognitivismus/Anti-Kognitivismus zu berücksichtigen. Auch mögliche Anknüpfungspunkte bei Alteritäts- (Levinas) oder Care-Ethiken werden zwar eingangs angekündigt (299), bleiben aber letztlich unberücksichtigt. Nicht zuletzt hätte sich für eine mögliche Lösung des auch bei Peucker angesprochenen Problems, wie nicht-objektivierende (Wert-)Akte Objektivität von Werten verbürgen können (310), eine mögliche Differenzierung angeboten, nämlich jene zwischen dem intentionalen Zugang zu objektiven Werten durch nicht-objektivierende Akten einerseits und dem konstitutiven Problem, worin die Objektivität bzw. Allgemeingültigkeit von Werten fundiert ist andererseits. Der Aufsatz schließt, entlang einer knappen Diskussion moralischer Dilemmata, wie dem vielzitierten TrolleyProblem, jedenfalls mit einer anderen interessanten und nicht minder überraschenden existenzialistischen Volte, bei der ein Sartre'scher Dezisionismus mit Husserls Idee eines „Rufes“ absoluten Sollens, welcher den Akteur affiziert und dessen Übernahme die Verantwortlichkeit seines Handelns konstituiert, kurzgeschlossen wird.

Auf die Analyse passiver und aktiver bzw. objektivierender und nicht-objektivierender Komponenten von Willens- und Gefühlsakten und deren „Überlagerungen“ fokussiert auch der abschließende Beitrag Vongehrs, „Husserls Studien zu Gemüt und Wille“. Vongehr, Mitherausgeber des von Husserl-Forschern lang erwarteten und als letzten Husserliana-Band projektierten Forschungsmanuskriptkonvoluts Studien zur Struktur des Bewusstseins, verortet dabei sowohl editionsgenetisch als auch methodologisch Husserls monumentales Projekt einer Klärung des Verhältnisses von transzendental-phänomenologischer und deskriptiv-psychologischer Bewusstseinstheorie. Thematisch gesehen, geht er dann den komplexen Fundierungsgeflechten zwischen axiologischen, volitiven und emotionalen Akten innerhalb der Sphäre praktischer Intentionalität und dem dabei leitenden Handlungsbegriff nach (344f.). Der Beitrag wird abgerundet mit einer konzisen, kritischen Diskussion der zentralen Binnendifferenzierung innerhalb der Gemütssphäre, nämlich jener zwischen intentionalen Gefühlsakten und nicht-intentionalen Gefühlsempfindungen. Husserls diesbezügliche Analysen verdienen umso mehr Aufmerksamkeit, als sich hier wichtige Bausteine für eine fällige phänomenologische Auseinandersetzung mit der gegenwärtig vieldiskutierten Cognitive Phenomenology- (vgl. Bayne and Montague 2011) bzw. Phenomenal Intentionality-Debatte (vgl. Kriegel 2013) in der analytischen Philosophie des Geistes finden ließen, auf die Vongehr bedauerlicherweise ebenso wenig eingeht 
wie die anderen AutorInnen des Bandes. Vongehr gewährt jedenfalls insgesamt einen guten ersten Einblick in jenen Husserliana-Band, der nicht nur der krönende Abschluss jahrzehntelanger Editionsforschung sein sollte, sondern gewiss neuen Generationen von Husserl-scholars reichlich Material für die phänomenologische Evaluierung einer ganzen Reihe aktueller Problemfelder bieten wird, was eine ehebaldige Publikation nur umso wünschenswerter macht.

Obwohl die Beiträge in ihrer Qualität zum Teil recht stark variieren, demonstrieren sie doch alle klar das kritische Potenzial und den konzeptuellen Reichtum des Husserl'schen Euvres für die Analyse zahlreicher aktueller Forschungsfragen. Und obwohl eine Auseinandersetzung mit einigen brisanten aktuellen Debatten, bei denen Husserl ebenso als ernstzunehmender Diskussionspartner präsentiert hätte werden können, fehlen (neben den erwähnten wäre hier etwa auch die besonders heiß diskutierte Extended-Mind-These oder auch die Diskussion um die Normativität der Intentionalität zu nennen), stellt der Sammelband insgesamt ein besonders gelungenes Beispiel für eine zeitgemäße Husserl-Forschung dar, die eben nicht nur philologisch-historische oder apologetische Interessen befriedigt.

\section{Referenzen}

Bayne, T., \& Montague, M. (Eds.). (2011). Cognitive Phenomenology. Oxford: Oxford University Press. Beyer, C. (1996). Von Bolzano zu Husserl: Eine Untersuchung über den Ursprung der phänomenologischen Bedeutungslehre. Dordrecht et al.: Kluwer.

Beyer, C. (2000). Intentionalität und Referenz: Eine sprachanalytische Studie zu Husserls transzendentaler Phänomenologie. Paderborn: Mentis.

Centrone, S. (Ed.). (2013). Versuche über Husserl. Hamburg: Meiner.

Chelstrom, E. (2013). Social Phenomenology: Husserl, Intersubjectivity and Collective Intentionality. Plymouth: Lexington.

Embree, L., \& Nenon, T. (Eds.). (2013). Husserl's Ideen. Dordrecht: Springer.

Frank, M., \& Weidtmann, N. (Eds.). (2010). Husserl und die Philosophie des Geistes. Berlin: Suhrkamp.

Ierna, C., Jacobs, H., \& Mattens, F. (Eds.). (2010). Philosophy, Phenomenology, Sciences: Essays in Commemoration of Edmund Husserl. Dordrecht: Springer.

Kriegel, U. (Ed.). (2013). Phenomenal Intentionality. Oxford: Oxford University Press.

Moran, D., \& Szanto, T. (Eds.) (in preparation). Discovering the 'We'. The Phenomenology of Sociality. O’Brien, L., \& Soteriou, M. (Eds.). (2009). Mental Action. Oxford: Oxford University Press.

Salice, A., Schmid, H. B. (Eds.) (forthcoming). Social Reality: The Phenomenological Approach. Dordrecht: Springer.

Schmid, H. B. (2000). Subjekt, System, Diskurs. Edmund Husserls Begriff transzendentaler Subjektivität in sozialtheoretischen Bezügen. Dordrecht et al.: Kluwer. 